\title{
Density Distribution of the Expanding Products of Steady-State Detonation of TNT
}

\author{
É. R. Pruuél, ${ }^{1}$ L. A. Merzhievskii, ${ }^{1}$ K. A. Ten, ${ }^{1}$ \\ P. I. Zubkov, ${ }^{1}$ L. A. Luk'yanchikov, ${ }^{1}$ B. P. Tolochko, ${ }^{2}$
}

A. N. Kozyrev, ${ }^{3}$ and V. V. Litvenko ${ }^{3}$

UDC 534.222 .2

Translated from Fizika Goreniya i Vzryva, Vol. 43, No. 3, pp. 121-131, May-June, 2007.

Original article submitted May 17, 2006.

This paper gives a description of a high-speed X-ray tomographic technique and the results of its application to finding the density distribution of detonation products of condensed explosives from measurements using synchrotron radiation. The data obtained for a cylindrical charge of pressed TNT of diameter 12.5-mm and density $1.6 \mathrm{~g} / \mathrm{cm}^{3}$ are compared with the results of solution of the corresponding gas-dynamic problem of steady-state detonation. The experimental and computational results are in satisfactory qualitative and quantitative agreement. The features of the employed technique and the distribution obtained are analyzed. The technique as a whole and the results obtained can be used to test and refine the known equations of state for detonation products and to construct new ones.

Key words: explosives, synchrotron radiation, detonation, density distribution

\section{INTRODUCTION}

Although a considerable body of information has been accumulated, steady-state detonation and the expansion of explosion products are still a subject of extensive experimental research. This is motivated by both the development of new explosives (HEs) and the need to refine available data on the characteristics of conventional HEs and eliminate ambiguity in their interpretation. Data on the expansion of detonation products are used to construct shock adiabats and to determine the coefficients of the widely-used semiempirical equations of state and kinetics of detonation transformation. A wide range of experimental techniques based on various physical principles has been designed. Studies, as a rule, have been performed with plane onedimensional detonation waves. Al'tshuller et al. [1] pro-

\footnotetext{
${ }^{1}$ Lavrent'ev Institute of Hydrodynamics, Siberian Division, Russian Academy of Sciences, Novosibirsk 630090; pru@hydro.nsc.ru; merzh@hydro.nsc.ru.

${ }^{2}$ Institute of Solid State Chemistry and Mechanochemistry, Siberian Division, Russian Academy of Sciences, Novosibirsk 630090.

${ }^{3}$ Novosibirsk State University, Novosibirsk 630090.
}

posed a conditional division of all methods for studying detonation processes into "external" and "internal." Without a detailed characterization of all the methods used, we note that the most correct results are provided by "internal" nonperturbing methods (which do not introduce perturbations into the processes studied), among which X-ray techniques appear to provide the fullest information.

$\mathrm{X}$-ray techniques have long and extensively been used to study the flow of detonation products [2-5]. Both perturbing (in X-ray measurements of the displacement of foils placed in HEs) and nonperturbing methods (in direct X-ray photography) have been implemented. One of the parameters that can be studied by such methods is the density distribution in the flow studied.

Usually, the exposure duration is fractions of a microsecond and the spatial resolution is about $1 \mathrm{~mm}$. The divergence of X-ray beams produced by point sources introduces additional difficulties into the interpretation of the data obtained. Molitoris [6] employed improved sources for X-ray generation, which provided an increased temporal resolution of up to $25 \mathrm{nsec}$ and a spatial resolution of up to $0.1 \mathrm{~mm}$. 
Advanced elementary particle accelerators produce beams of various natures with a high penetration power and unique spatial-temporal characteristics, including nearly parallel beams. Therefore, using these facilities is a promising direction for the fundamental improvement of the spatial-temporal characteristics of the nonperturbing diagnostic techniques for studying hydrodynamic flows. In studies of shock-wave and explosive processes in condensed media, the high penetration power of beams allows, in some cases, the internal characteristics of flow to be determined using tomographic methods.

Of the possible radiation-based approaches to detonation research, two methods - proton-beam and synchrotron-radiation techniques - are now at the stage of implementation $[7,8]$. The first experiments conducted with the use of proton beams are described in $[9,10]$. They demonstrated the possibility of using proton radiography to study processes in condensed explosives. Much more progress has been made in the investigation of detonation and shock-wave processes using synchrotron radiation [8, 11-13]. A number of problems of steady-state detonation, the dynamics of formation of diamond particles, and deformation and failure of inert media have been solved.

The present paper describes a synchrotron radiation method and the results of using this method in tomographic studies of the density distribution of the expanding detonation products of condensed HEs. The data obtained are compared with the results of solution of a two-dimensional steady-state problem of detonation product expansion.

\section{EXPERIMENTAL SETUP}

Synchrotron radiation (SR) allows one to implement a nonperturbing internal method for measuring the spatial density distribution of detonation products of condensed HEs. The synchrotron radiation technique for studying detonation and shock-wave processes is set out in detail in [8]; therefore, we shall only describe the experimental setup. The employed setup (Fig. 1) allows one to use the principles of X-ray tomography and reconstruct the density distribution in detonation products.

We studied the detonation products of cylindrical charges of pressed TNT of diameter $12.5 \mathrm{~mm}$ and density $1.6 \mathrm{~g} / \mathrm{cm}^{3}$, initiated at one end with a standard detonator. The charges had a length of $60 \mathrm{~mm}$, sufficient to consider the detonation process steady-state.

The measurement region (the plane perpendicular to the charge axis) was probed with a line-focus SR

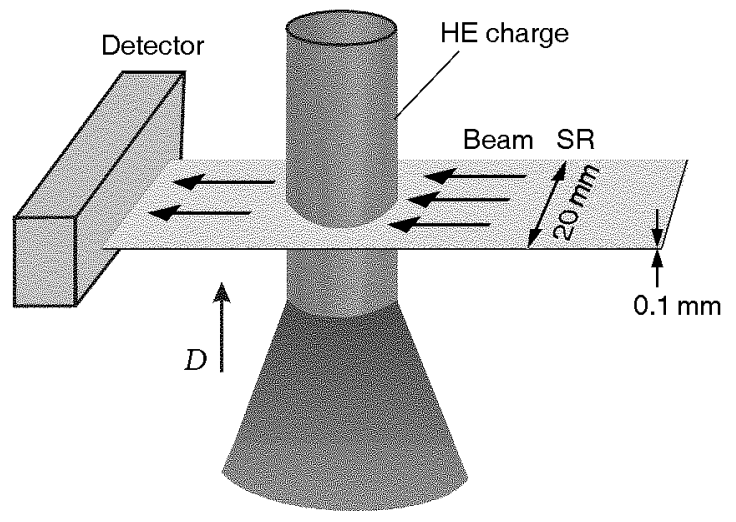

Fig. 1. Experimental setup.

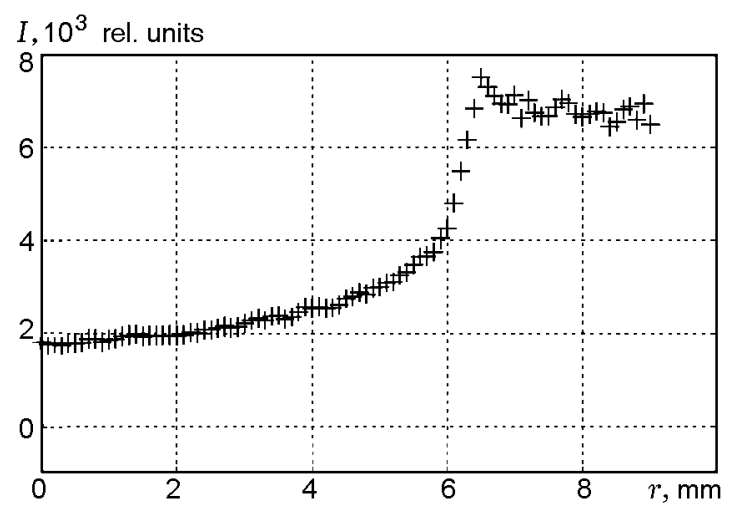

Fig. 2. Radial distribution of the radiation intensity transmitted through the sample.

beam - a beam of X-ray nonmonochromatic radiation with a flash time of $10^{-9}$ sec and a hardness of 10-30 keV. The quantity to be measured was the intensity of the transmitted SR beam attenuated by interaction with the material. The radial distribution of the transmitted beam intensity was measured with a DIMEX linear microstrip gas detector (see Fig. 1), which consists of 256 sensing elements spaced $0.1 \mathrm{~mm}$ apart and has the ability to store successive 32 frames at $0.5 \mu \mathrm{sec}$ intervals with an exposure time of $\approx 1 \mathrm{nsec}$. Thus, the result of one experiment is an X-ray streak "cinema" of duration $16 \mu \mathrm{sec}$ with a spatial width of the tested region of $\approx 25 \mathrm{~mm}$.

\section{DETERMINING THE AMOUNT OF MATERIAL ON THE BEAM FROM DETECTOR DATA}

Traditionally, to obtain a tomographic image of an object, one needs to perform a survey of an object at several aspect angles. The use of charges of cylindrical 


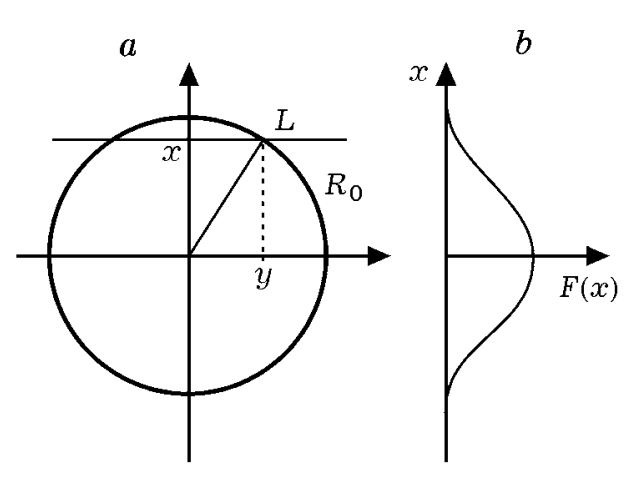

Fig. 3. Diagram of processing of records of the radiation transmitted through the sample $(x$ and $y$ are the coordinates along and across the beam).

shape provided axial symmetry of the detonation product flow, which allowed the radial density distribution in the observed cross section of the charge to be reconstructed using information obtained from just one aspect angle. Under the assumption of steady-state flow, the total density distribution of the detonation products is reconstructed [the function $\rho(r, z)$ is plotted, where $r$ and $z$ are the radial and axial coordinates].

The results of the experiments are distributions of the radiation intensity transmitted through the tested sample at various times. An example of the distribution is shown in Fig. 2. Using these data, it is required to determine the radial dependence of the density of the expanding detonation products at a particular cross section. Generally, the intensity attenuation is determined by the multifactor interaction of the radiation with the material, i.e., by the dependence of the absorption coefficient on wavelength, elastic and inelastic scattering, ionization losses, bending of the beam path on the charge-air boundary and inside the tested sample at density gradients, etc. The result is additional affected by the nonlinearity of the detector and its complex spectral sensitivity. As a result, even if all these factors are incompletely taken into account, the energy recorded by the detector from the beam $l$ transmitted through an object is given by the relation

$$
J_{l}=\int_{E} D(E) J_{0 l}(E) \exp \left(-\int_{l} \mu(r, E) d r\right) d E,
$$

where $E$ is the X-ray energy, $J_{0 l}(E)$ is the X-ray source energy density along the beam $l, D(E)$ is the coefficient of absorption of radiation with energy $E$ by the detector, and $\mu(r, E)$ is the linear coefficient of absorption of radiation with energy $E$ at the point $r$. For a further simplification of the problem, it is assumed that the degree of attenuation of the transmitted radiation depends only on the integral of the density over the seg- ment occupied by the explosion products and that the beam propagates in a straight line. Then, introducing the coordinates $x$ and $y$ in accordance with Fig. 3a, we have

$$
\begin{gathered}
\ln J_{0 l}-\ln J_{l}(x, \lambda) \\
=a(\lambda) \int_{-\sqrt{R_{0}^{2}-x^{2}}}^{\sqrt{R_{0}^{2}-x^{2}}} \rho\left(\sqrt{x^{2}+y^{2}}\right) d y \\
=2 a(\lambda) \int_{0}^{\sqrt{R_{0}^{2}-x^{2}}} \rho\left(\sqrt{x^{2}+y^{2}}\right) d y,
\end{gathered}
$$

where $a(\lambda)$ is the absorption coefficient at wavelength $\lambda$ ( $a=$ const for monochromatic radiation). Given the function $a(\lambda)$, the problem can be reduced to solving the equation

$$
F(x)=\int_{-\sqrt{R_{0}^{2}-x^{2}}}^{\sqrt{R_{0}^{2}-x^{2}}} \rho\left(\sqrt{x^{2}+y^{2}}\right) d y,
$$

where the function $F(x)$ (see Fig. 3b) is related to $J_{0 l}(\lambda), J_{l}(x, \lambda)$, and $a(\lambda)$ in an obvious manner. The change of variables $r=\sqrt{x^{2}+y^{2}}$ reduces the equation to the form

$$
F(x)=\int_{x}^{R_{0}} \rho(r) \frac{2 r}{\sqrt{r^{2}-x^{2}}} d r
$$

where $\rho(r)$ is the required dependence of the density on the radius and $F(x)$ is the experimentally measured shadow from a cylinder of radius $R_{0}$ (see Fig. 2 ). It was shown [14] that by further transformations, the solution of the problem of reconstructing the radial density distribution is given by the formula

$$
\rho(r)=\frac{1}{\pi r} \frac{d}{d r} \int_{r}^{1} \frac{x F(x)}{\sqrt{x^{2}-r^{2}}} d x .
$$

Thus, under the assumptions formulated above, the solution of the density reconstruction problem involves no difficulties if $F(x)$ is a smooth differentiable function. In fact, the experiment provides values of $F(x)$ in a discrete set of points, and these values are determined at a certain inaccuracy. Especially large errors in the solution of Eq. (2) using experimental data are due to inaccuracies in determining the derivative of $F(x)$ in $(2)$. Since the resulting distribution $\rho(r, z)$ depends greatly on particular values of $F(x)$ and its derivative, an accurate reconstruction of this distribution requires the development and implementation of complex algorithms for regularizing the experimental data array and 


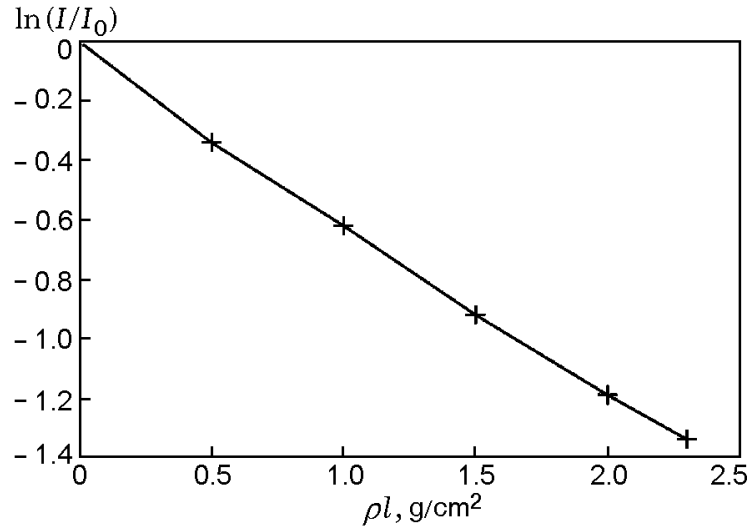

Fig. 4. Calibration curve for determining the amount of the radiographed material.

solving the corresponding inverse problem. A relatively simple computational-experimental method for solving the problem in two stages was proposed to simplify the reconstruction procedure. In the first stage, we determined the mass of the material on the beam path rather than the density. We used the above-mentioned assumption that the degree of radiation attenuation depended only on the mass of the material tested, i.e., we assumed that

$$
F(x)=f\left(\int_{0}^{L} \rho(y) d y\right),
$$

where the unknown function $f$ was determined experimentally. From the data of the experiments, we plotted the dependence of the recorded intensity on the amount of the material (TNT) for each channel of the detector. Homogeneous plates of various thicknesses (3-5 measurements) were radiographed. The results of the determination of the function are shown in Fig. 4. The experimental data on the plane $\left\{\ln \left(I / I_{0}\right)\right.$, $\left.\rho l\right\}$ are approximated well by the parabola

$$
\ln \frac{J_{l}}{J_{0 l}}=-0.66 \rho l+0.03(\rho l)^{2} .
$$

As a first approximation, the obtained dependence can be considered linear with good accuracy. The calibration curve plotted in this manner was used to determine the amount of the material on the beam based on the measured transmitted intensity. The result of determination of $\rho l$ using the data of Fig. 2 is shown in Fig. 5. By performing measurements in various cross sections of the detonating charge, one obtains the complete spatial distribution of $\rho l=\rho l(r, z)$ using the method described above. Such a surface for one of the experiments conducted is shown in Fig. 6.

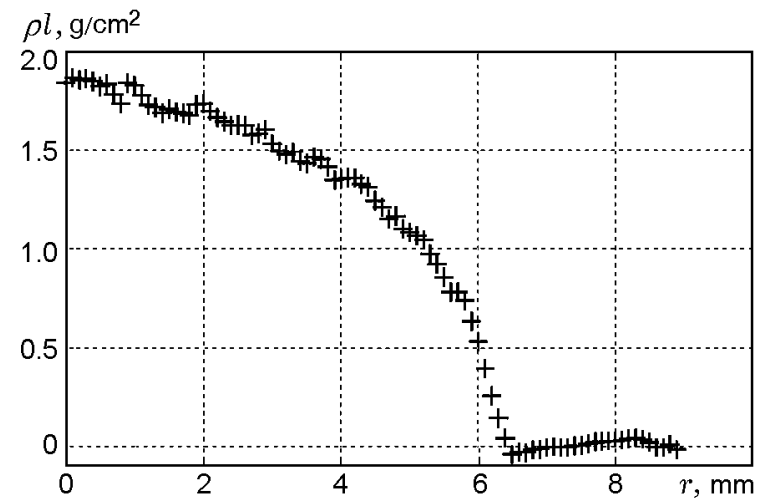

Fig. 5. Radial distribution of the amount of the radiographed material reconstructed from the calibration dependence.

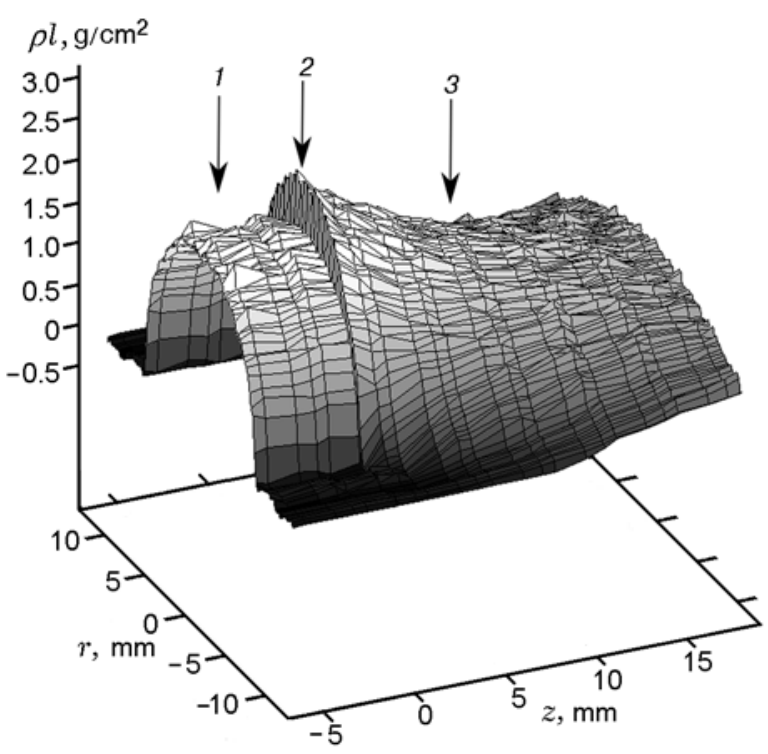

Fig. 6. Spatial distribution of the amount of the radiographed material: 1) unreacted HE; 2) detonation front; 3) detonation products.

\section{RECONSTRUCTING THE SPATIAL DENSITY DISTRIBUTION}

In the second stage, the distribution $\rho l(r, z)$ was reconstructed from the curves of $\rho(r, z)$. According to the general concepts of the gas dynamics of detonation product expansion, the distribution of the parameters of the products should be described by smooth functions, and, hence, they can be are approximated by some appropriate functions, in which the free parameters are calculated so as to obtain the best fit to experimental data. From the aforesaid, the distribution $\rho(r, z)$ was 

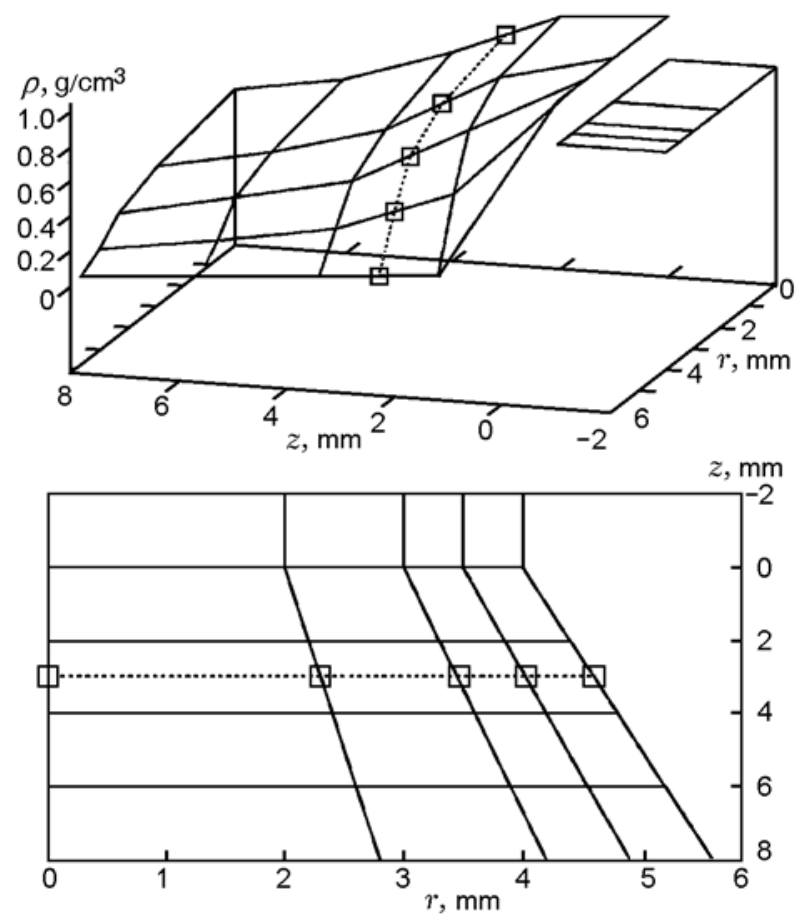

Fig. 7. Interpolation grid for density reconstruction: the squares indicate the points lying on the same beam $(z=$ const $)$.

sought in the form of a cubic spline drawn through the nonuniformly arranged nodes of a grid which was made finer to the maximum radius of the expansion (Fig. 7). The varied parameters are the density at each node, the initial radius of the charge, and the maximum angle of expansion of the detonation products in the system of the front.

The grid density on the space and time coordinates was such that the number of nodes was sufficient for a good description of the experimental dependences $\rho l(r, z)$ and the solution obtained was sufficiently smooth. Grids with a number of parameters 20-40 were used. The parameters were chosen by minimizing the standard deviations of the calculated values of $\rho l(r, z)$ from experimental values. The minimum of the function of standard deviations was sought numerically. The results of the density distribution reconstruction are shown in Fig. 8. Figure 8b and c gives geometrical images of the same function $\rho(r, z)$ in two aspects, and Fig. 8 a gives the projection of the same surface onto the spatial plane. The last image is equivalent to an instantaneous X-ray photograph of the detonating priming charge obtained using the conventional X-ray technique, e.g., in $[4,5]$. In the photograph in Fig. 8a, one can see the distinct boundary of the expanding products and the trajectories of lateral rarefaction waves.
An advantage of the given method is that the function describing the spatial density distribution can be constructed by using information from different time layers and by combining data of different experiments. In particular, the combination of the data of three experiments in one of the cases increased the reliability of the recorded parameters from a statistical point of view and improved their temporal resolution. The properties of the constructed distribution function allow it to be extrapolated to some region of coordinate values located beyond the region of experimental observation.

The proposed technique provides information on the density distribution in the region of detonation transformation and product expansion; however, the current capabilities of beam generation and the existing detector parameters do not allow reliable data on the chemical reaction zone to be obtained. Measuring the density distribution in the chemical reaction zone was a subject of a separate study. The temporal resolution along the charge axis obtained using a combination of data from several experiment was estimated to be $\approx 0.2 \mu \mathrm{sec}(1.4 \mathrm{~mm})$. The spatial resolution along the radius corresponds to the step of the grid used for density reconstruction. The boundaries of the charge and the region of detonation product expansion were determined with an accuracy of $\approx 0.2 \mathrm{~mm}$. The accuracy of density determination was $\pm 0.2 \mathrm{~g} / \mathrm{cm}^{3}$, and at low gradients and large statistics, it increased to $\pm 0.1 \mathrm{~g} / \mathrm{cm}^{3}$.

\section{PROBLEM OF EXPANSION OF STEADY-STATE DETONATION PRODUCTS}

We consider the problem of the expansion of steady-state detonation products from the end of a cylindrical HE charge in vacuum, which was solved earlier with the aim of studying the asymptotic behavior of the parameters of the products [15]. We ignore the finiteness of the reaction zone and the real curvature of the detonation front (the Chapman-Jouguet surface) and assume that the Chapman-Jouguet surface is planar up to the boundary of the charge. From a gasdynamic point of view, the formulation of this problem is equivalent to the problem of supersonic jet flow.

In a coordinate system moving at the detonation velocity, the flow is described by the equations

$$
\begin{gathered}
\frac{\partial v}{\partial z}-\frac{\partial u}{\partial r}=0, \\
\frac{\partial}{\partial r}(r \rho v)+\frac{\partial}{\partial z}(r \rho u)=0, \\
u^{2}+v^{2}+2 h(\rho)=\text { const }=U^{2},
\end{gathered}
$$




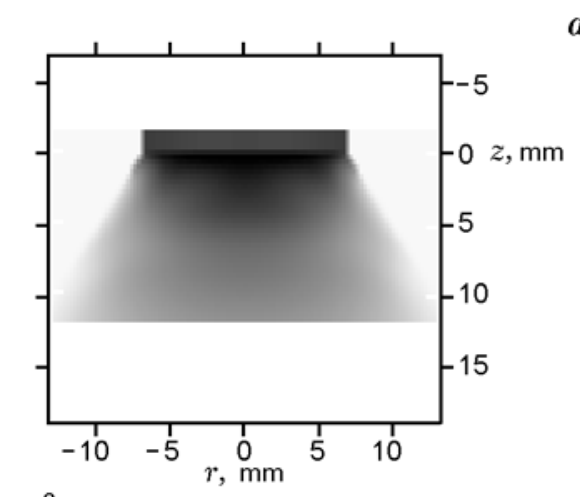

$a$
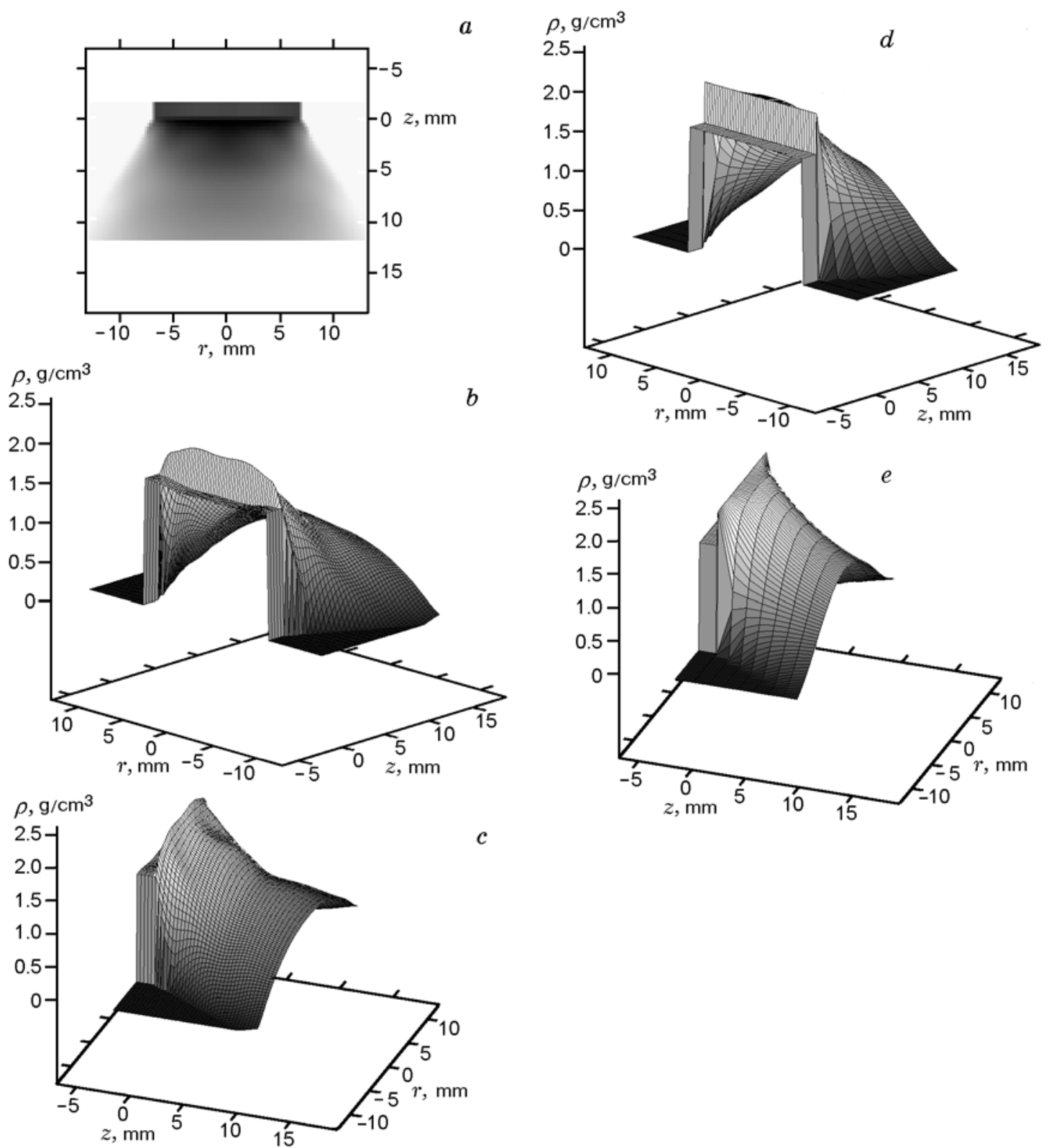

$c$

Fig. 8. Spatial density distribution of detonation products: experiment $(\mathrm{a}-\mathrm{c})$ and calculation $(\mathrm{d}$ and $\mathrm{e})$.

where $z$ and $r$ are the longitudinal and radial coordinates, $u$ and $v$ are the corresponding velocity components, $h(\rho)$ is the enthalpy, and $U$ is the maximum expansion velocity. The computation region is shown in Fig. 9, where $A O$ is the sonic surface, $A B$ is the limiting streamline, and $O z$ is the symmetry axis. The boundary conditions are as follows: for $z=0$ and $0 \leqslant r \leqslant r_{0}$, $u=D, v=0$, and $\rho=\rho_{\mathrm{CJ}}$; for $z>0$ and $r=0$, $v=0$. In the neighborhood of the point $z=0, r=r_{0}$, Prandtl-Mayer flow occurs.

The detonation products are assumed to be an ideal polytropic gas with a constant polytropic exponent $\gamma$. In this case, the limiting streamline $A B$ is a straight line directed at the angle

$$
\Theta_{\lim }=\frac{\pi}{2}\left(\sqrt{\frac{\gamma+1}{\gamma-1}}-1\right) .
$$




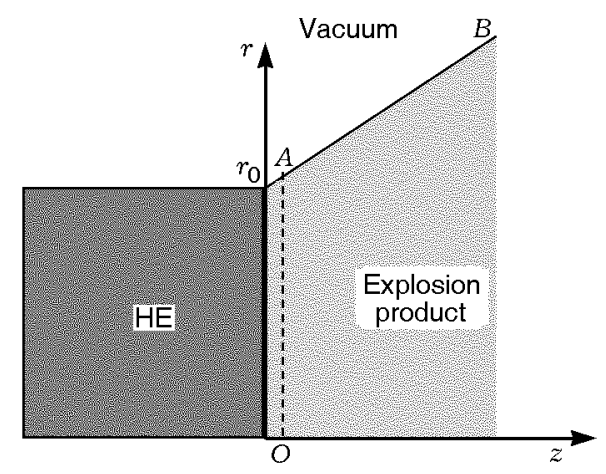

Fig. 9. Computation region for the problem of expansion of detonation products.

To solve the formulated problem of supersonic gas flow, it is necessary, using the initial data specified on the sonic surface (Chapman-Jouguet plane), to calculate the flow parameters in a certain neighborhood $A O$ belonging to the region considered. In the neighborhood of the point $z=0, r=r_{0}$, the sought solution should be joined to the solution of the Prandtl-Mayer problem. Both preliminary steps in the calculations were performed using the method described in [15]. The primary problem was solved numerically using a method of characteristics. As an example, the calculation results for $\gamma=3.1$ in the form of the surface $\rho(r, z)$ are shown in Fig. 8d and $\mathrm{f}$ (the same aspect angle as for the experimental surfaces).

\section{DISCUSSION OF RESULTS}

In the experiments performed, in addition to gathering data required to find the density distribution, we recorded the evolution of the detonation wave profile. This made it possible to determine the detonation propagation velocity $D$. It was found that $D=$ $6.95 \pm 0.05 \mathrm{~km} / \mathrm{sec}$. As noted above, the implementation of the method does not allow the resolution of the flow parameters in the chemical transformation zone. The adiabatic exponent calculated from the maximum value of the experimentally measured density of the products, which was set equal to the density in the ChapmanJouguet plane, was $\gamma=2.9-3.2$. Both values agree with the data for pressed TNT for the same initial density (see, e.g., $[16,17])$.

Figure $8 \mathrm{a}-\mathrm{c}$ gives data on the experimental density distribution in the form of the surface $\rho(r, z)$ and the projection of this surface onto the plane $(r, z)$. Calculated dependences $(r, z)$ are given in Fig. 8d and e. Despite the simplified formulation of the gas-dynamic

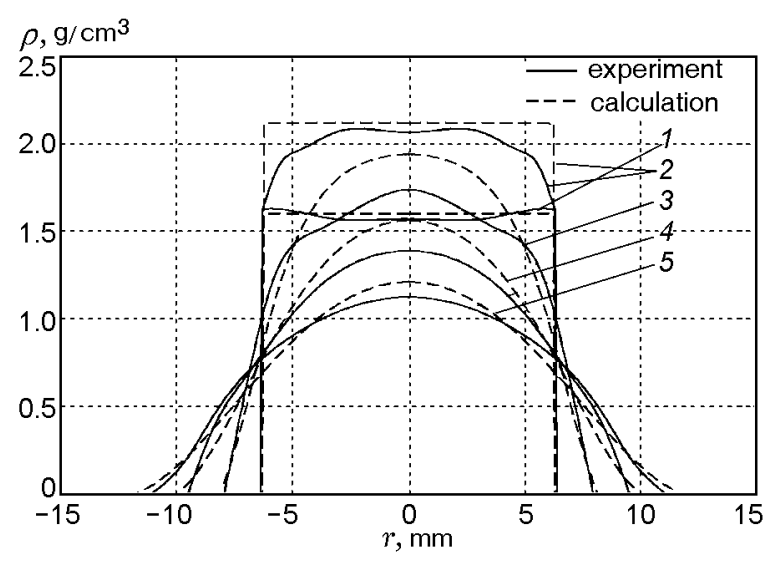

Fig. 10. Comparison of experimental and calculated density distributions along the radius at various distances $l$ from the detonation front: $l=-2.4$ (unreacted $\mathrm{HE}$ ) (curves 1), 0 (detonation front) (curves 2), 2.4 (curves 3), 4.8 (curves 4), and $7.2 \mathrm{~mm}$ (curves 5 ).

problem (the assumption of the adiabaticity of detonation products with a constant adiabatic exponent), a visual comparison of the experimental and calculated data shows that they are in good agreement. A more correct quantitative comparison is performed in Fig. 10 and 11. The first figure gives radial density distributions at various fixed distances from the detonation front. The experimental data differ from the computational results by not more than $10 \%$, which can be considered satisfactory, especially when taking into account the approximate formulation of the problem being solved. At the same time, we note that near the boundaries of the charge, the behavior of the experimental dependences differ from the calculated ones. The experimental curves show a certain increase or decrease in the rate or density decay in this region. This seems to be related to the departure of the radiation from the initial path at the edge of the charge and not to the real nonmonotonic density distribution. This feature of the employed technique is reflected on distributions of the transmitted radiation for radiography of the cylindrical charge which has not yet detonated (1 in Fig. 10). This explanation of the observed effect is supported by the fact that it decreases and almost completely disappears as the radius of expansion of the products increase.

In Fig. 11, the calculated and experimental density distributions along the charge axis are compared for various fixed values of the radius. Distributions along this coordinate appear in the solutions of one-dimensional problems used to estimate the parameters of expanding detonation product [18-20]. The data in Fig. 11a distributions of the parameters on the charge axis are the most comparable to such one-dimensional solu- 

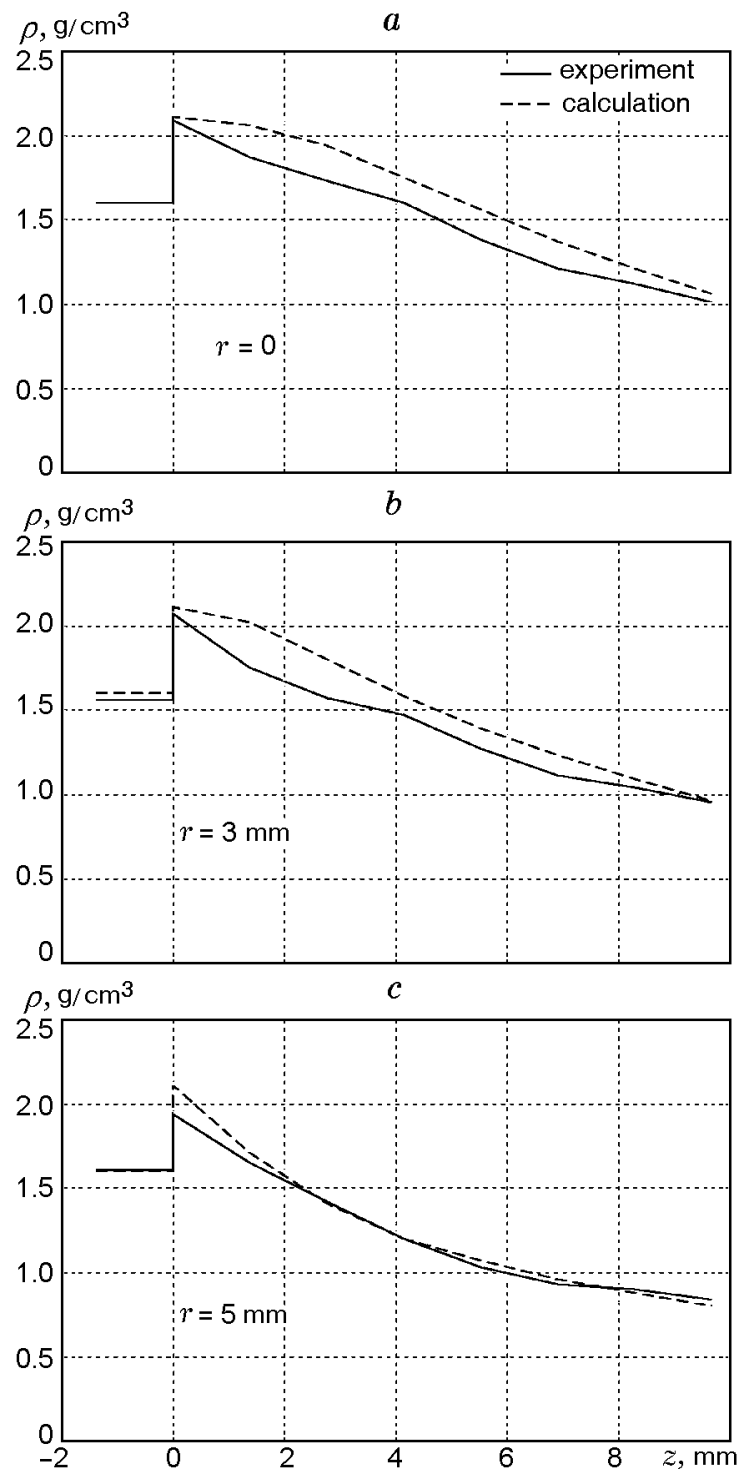

Fig. 11. Comparison of the experimental and calculated density distributions along the longitudinal coordinate.

tions. According to the classical detonation model, the steady-state chemical reaction zone of a plane detonation wave is followed by a self-similar rarefaction wave $[18,21]$. In this case, the curves given in Fig. 9a should be nearly linear [linear for $\gamma=3$ and exponential with an exponent of $2 /(\gamma-1)$ for the other values of $\gamma$ ]. The calculated curve is indeed almost linear beginning at $z>2 \mathrm{~mm}$. The experimental curve is in worse agreement with a linear density distribution along the coordinate.

As was found in the experiments, the real steadystate detonation parameters referred to the ChapmanJouguet plane and the width of the chemical reaction

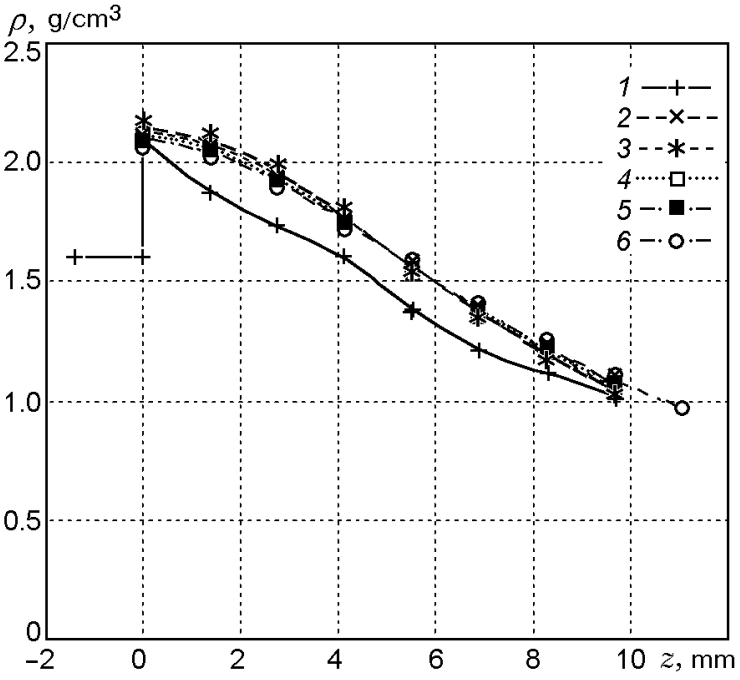

Fig. 12. Comparison of experimental and calculated density distributions along the $z$ axis: points 1 refer to the experiment and points $2-6$ refer to calculations for $\gamma=2.9,3.0,3.1,3.2$, and 3.3 , respectively.

zone can vary widely depending on experimental conditions [3]. An analysis leads to the conclusion that the detonation wave structure postulated in the detonation model can be reached only asymptotically. In practice, an unsteady process occurs, in which the width of the chemical reaction zone is not constant and the Chapman-Jouguet plane moves relative to the shock. It has also been shown [22] that the effect of the unsteadiness of the chemical reaction zone on the detonation product flow parameters is limited by a region of the order of about five characteristic sizes of the zone. For the HE used in the experiments considered, the chemical reaction zone is $\approx 1 \mathrm{~mm}$ [16]. These circumstances may influence the nature of the axial distribution observed in the experiments since the region considered is close to the reaction zone. At $r=3 \mathrm{~mm}$, the experimental and calculated curves for $z>4 \mathrm{~mm}$ are much closer to each other, and at $r=5 \mathrm{~mm}$, they almost coincide even for $z>2 \mathrm{~mm}$. Apparently, an increase in the degree of unloading of the products leads to an increase in the degree of their equilibrium and agreement with the adiabatic approximation. The above-mentioned edge effect makes an additional contribution to the behavior of the experimental curve. At the same time, the deviation from self-similarity becomes more and more considerable.

The systematic deviation of the calculated curves from the experimental curves is irrelevant to the choice of a particular value of $\gamma$ for calculation. This follows from a comparison of the experimental density distributions with the calculated ones obtained for various 
values of $\gamma$ from the above-mentioned range. This is illustrated by Fig. 12, which gives density distributions along the axis calculated for various values of $\gamma$. The results for $r \neq 0$ are similar.

In real experiments, including ours, the non-onedimensional nature of the process plays a significant role in the distribution of the parameters of the expanding products. In this case, it should be taken into account that in addition to the radial expansion under the action of the rarefaction wave propagating from the lateral surface of the charge, another factor that influences the distribution of the parameters is the detonation-front curvature intrinsic in charges of finite diameter, especially in charge of relatively small diameter.

We note that to refine and derive the equations of state for detonation products, one does not need to use the parameters in the region influenced by the nonstationary nature of the chemical reaction zone; the more so as these equations link characteristics in the state of thermodynamic equilibrium. In this connection, the determination of the range of equilibrium parameters should be an important point in the analysis and interpretation of the data obtained.

The tomographic reconstruction of the density distribution of the detonation products became possible due to the symmetry of the process. In practice, there are many factors that violate the flow symmetry. The main of them are apparently the inhomogeneity of the charges used and the instabilities due to the expansion of detonation products. The extent to which these factors influence the accuracy of reconstructing the density dynamics has not been completely clarified, but the results of the study demonstrate that the density distribution was reconstructed with reasonable accuracy.

\section{CONCLUSIONS}

A technique implementing the capabilities (described in [8]) of synchrotron radiation for studying explosive and shock-wave processes was described. It uses X-ray tomography principles and involves a twostage reconstruction of the density distribution of the expanding products of steady-state detonation with the construction of a calibration curve describing radiation absorption by the material. This method was employed to construct a spatial density distribution function for the detonation products of TNT with an initial density of $1.6 \mathrm{~g} / \mathrm{cm}^{3}$. The corresponding gas-dynamic problem is solved in an adiabatic approximation. A comparison of the results of the experiments with calculated data showed that their were in satisfactory agreement in a region fairly close to the detonation front. At the same time, systematic differences were observed, which might be caused by the neglect of the detonation-front curvature in the calculation and the real nonequilibrium of the products due to the effect of the chemical reaction zone, whose existence was indicated in [22].

The technique as a whole and the results obtained can be used to test and refine known equations of state of detonation products and construct new ones. It should be noted that the method is suitable for studies of the behavior of low-density inert media under shockwave loading, such as those studied in the experiments of, e.g., [23].

This work was supported by the Integration project of the Siberian Division of the Russian Academy of Sciences No. 120, Youth project of the Siberian Division of the Russian Academy of Sciences No. 18, Foundation "Leading Scientific Schools of Russian Federation" (Grant No. NSh-2073.2003.1), and Russian Foundation for Basic Research (Grant Nos. 05-03-32752 and 06-0217335).

\section{REFERENCES}

1. L. V. Al'tshuler, G. S. Doronin, and V. S. Zhuchenko, "Detonation regimes and Jouguet parameters of condensed explosives," Combust., Expl., Shock Waves, 25, No. 2, 209-224 (1989).

2. W. C. Rivard, D. Venable, W. Fickett, and W. C. Davis, "Flash X-ray observation of marked mass points in explosive products," in: Proc. Fifth Int. Symp. on Detonation, Pasadena (1970), pp. 3-11.

3. V. V. Dorokhin, V. N. Zubarev, Yu. K. Orekin, et al., "Motion of explosion products behind a detonation wave front," Combust., Expl., Shock Waves, 21, No. 4, 471474 (1985).

4. V. A. Komrachkov, A. D. Kovtun, and Yu. M. Makarov, "Use of pulsed radiography in studies of shock-wave initiation of TATB," Combust., Expl., Shock Waves, 35, No. 2, 198-202 (1999).

5. K. N. Panov and V. A. Komrachkov, "Radiographic study of the evolution of the material density profile behind the front of a divergent shock wave in an explosive," Combust., Expl., Shock Waves, 40, No. 5, 591-596 (2004).

6. J. D. Molitoris, X-ray, Detonations, and Dead Zone, http://www-cms.llnl.gov/s-t/dead_zone_str.html.

7. N. S. P. King, E. Ables, K. Adams, et al., "An 800-MeV proton radiography facility for dynamic experiments," Nucl. Instrum. Meth. Phys. Res. A, 424, 84-91 (1999). 
8. A. N. Aleshaev, P. I. Zubkov, G. N. Kulipanov, et al., "Application of synchrotron radiation for studying detonation and shock-wave processes," Combust., Expl., Shock Waves, 37, No. 5, 585-593 (2001).

9. C. L. Mader, J. D. Zumbro, E. N. Ferm, et al., "Proton radiographic and numeric modeling of colliding, diverging PBX-9502 detonations," in: 12th Int. Detonation Symp., Snoumass, Colorado (2002).

10. E. N. Ferm et al., "Proton radiography experiments on shocked high explosive products," in: AIP Conference Proceedings, Vol. 706 (2004), pp. 839-842.

11. P. I. Zubko, G. N. Kulipanov, L. A. Lukyanchikov, et al., "Observation of compression and failure waves in PMMA by means of synchrotron radiation," Combust., Expl., Shock Waves, 39, No. 2, 240-242 (2003).

12. V. M. Titov, B. P. Tolochko, K. A. Ten, et al., "The formation kinetics of detonation nanodiamonds," in: D. M. Gruen et al. (eds.), Synthesis, Properties and Applications of Ultrananocrystalline Diamond, Proc. Conf.of the NATO ARW (St. Petersburg, Russia, June 7-10, 2004), Springer, Dordrecht (2005), pp. 169-180.

13. K. A. Ten, O. V. Evdokov, I. L. Zhogin, et al., "Measurements of the density distribution in a detonation wave using synchrotron radiation, preprint," Preprint, Budker Institute of Nuclear Physics, Sib. Div., Russian Acad. of Sci., Novosibirsk (2005).

14. K. A. Ten, V. M. Aul'chenko, O. V. Evdokov, et al., "Measurements of the density distribution for detonation of explosives by means of synchrotron radiations," in: Physics of Extreme Sates of Matter, Institute of Problems of Chemical Physics, Russian Academy of Sciences, Chernogolovka (2003), pp. 40-42.
15. L. A. Merzhievskii and V. A. Filimonov, "Asymptotic curve of the scattering of the products of a steady-state detonation, J. Appl. Mech. Tech. Phys., No. 2, 177-182 (1977).

16. A. N. Dremin, S. D. Savrov, V. S. Trofimov, and K. K. Shvedov, Detonation Waves in Condensed Media [in Russian], Nauka, Moscow (1970).

17. L. P. Orlenko (ed.), Physics of Explosion [in Russian], Fizmatlit, Moscow (2002).

18. Ya. B. Zel'dovich, "On the pressure and velocity distributions in detonation products, in particular, in a spherically propagating detonation wave," Zh. Éksp. Teor. Fiz., 12, No. 9, 389-406 (1942).

19. G. I. Taylor, "The dynamics of the combustion products behind plane and spherical detonation fronts in explosives," Proc. Roy. Soc. A, 200, 235-247 (1950).

20. G. T. Volodin, "Distribution of parameters of detonation products of condensed explosives," Combust., Expl., Shock Waves, 27, No. 1, 117-121 (1991).

21. Ya. B. Zel'dovich and A. S. Kompaneets, Theory of Detonation, Academic Press, New York (1960).

22. S. M. Bakhrakh, A. A. Evstigneev, V. N. Zubarev, and A. A. Shanin, "Effect of the finite rate of high-explosive decomposition on the determination of detonation parameters," Combust., Expl., Shock Waves, 17, No. 6, 685-688 (1981).

23. L. A. Merzhievskii, A. A. Evstigneev, V. N. Zubarev, and A. A. Shanin, "Synchrotron radiation study of the dynamics of shock-wave compression of an aerogel. Extreme states of matter. Detonation. Shock Waves," in: VII Chariton Readings, Proc. Int. Conf. Sarov (2005), pp. 665-669. 